\title{
On the spectra of certain integro- differential-delay problems with applications in neurodynamics
}

Article

Accepted Version

Grindrod, P. and Pinotsis, D. (2010) On the spectra of certain integro-differential-delay problems with applications in neurodynamics. Physica D: Nonlinear Phenomena, 240 (1). pp. 13-20. ISSN 0167-2789 doi:

https://doi.org/10.1016/j.physd.2010.08.002 Available at https://centaur.reading.ac.uk/15743/

It is advisable to refer to the publisher's version if you intend to cite from the work. See Guidance on citing.

To link to this article DOI: http://dx.doi.org/10.1016/j.physd.2010.08.002

Publisher: Elsevier

All outputs in CentAUR are protected by Intellectual Property Rights law, including copyright law. Copyright and IPR is retained by the creators or other copyright holders. Terms and conditions for use of this material are defined in the End User Agreement.

www.reading.ac.uk/centaur 
Central Archive at the University of Reading

Reading's research outputs online 


\title{
On the spectra of certain Integro-Differential-Delay problems with applications in neurodynamics
}

\author{
Peter Grindrod * and Dimitris Pinotsis *广
}

May 4, 2010

\begin{abstract}
We investigate the spectrum of certain Integro-Differential-Delay equations (IDDEs) which arise naturally within spatially distributed, nonlocal, pattern formation problems. Our approach is based on the reformulation of the relevant dispersion relations with the use of the Lambert function. As a particular application of this approach, we consider the case of the Amari delay neural field equation which describes the local activity of a population of neurons taking into consideration the finite propagation speed of the electric signal. We show that if the kernel appearing in this equation is symmetric around some point $a \neq 0$ or consists of a sum of such terms, then the relevant dispersion relation yields spectra with an infinite number of branches, as opposed to finite sets of eigenvalues considered in previous works. Also, in earlier works the focus has been on the most rightward part of the spectrum and the possibility of an instability driven pattern formation. Here, we numerically survey the structure of the entire spectra and argue that a detailed knowledge of this structure is important within neurodynamical applications. Indeed, the Amari IDDE acts as a filter with the ability to recognise and respond whenever it is excited in such a way so as to resonate with one of its rightward modes, thereby amplifying such inputs and dampening others. Finally, we discuss how these results can be generalised to the case of systems of IDDEs.
\end{abstract}

\footnotetext{
${ }^{*}$ Department of Mathematics and the Centre for Integrative Neuroscience and Neurodynamics, University of Reading, UK

${ }^{\dagger}$ Present address: Wellcome Trust Centre for Neuroimaging, University College London, UK
} 


\section{Introduction}

In this paper we consider a class of integro-differential-delay equations (IDDEs) that arise naturally within spatially distributed, non-local, pattern formation problems. As an immediate application we will show how these occur within the perturbation analysis of stationary states for a class of problems from neurodynamics. However the analysis presented here is quite general. In such problems the state of the system is represented by a state variable $u(\mathbf{x}, t)$ dependent upon time $t$, and location $\mathbf{x}$, ranging over some domain in $\mathbb{R}^{n}$. We will have a dynamical equation relating the rate of change of the state at any particular point to the present and past values of the state at all other points both locally and nonlocally. This nonlocal dependence results in an integral term with kernel combining the weighted influences from all other connected locations, as well as in an intrinsic transmission speed which in turn yields spatially dependent time delays.

Under the most simplifying of assumptions of spatial homogeneity and hence translational symmetry we will assume that a steady-state, time independent, solution, $u(\mathbf{x}, t)=u_{0}$ say, is known and that by linearising around it we may examine the stability behaviour of local solutions. Typically, for problems defined on an unbounded domian $\mathbf{x} \in \mathbb{R}^{n}$, we take the Fourier transform of the resulting linearised problem, writing $u(x, t)-u_{0} \sim \exp (\sigma t+i \mathbf{k} \cdot \mathbf{x})$. This results in an equation to be solved for the "dispersion relation", yielding the continuous spectrum, $\sigma=\sigma(\mathbf{k})$, as a function of the wave number.

The intrinsic nature of the IDDE is reflected within the structure of this equation and we will assume it is of the general form

$$
\sigma+\text { constant }=\mathcal{H}(\mathbf{k}, \sigma)
$$

where the $\sigma$ dependence of $\mathcal{H}$ includes a factor of the form $\exp (-\varepsilon \sigma a)$ for some constants $\varepsilon, a$. The equation is thus transcendental and the dispersion curve has solution branches of infinite multiplicity which reflects the infinite degrees of freedom introduced through the delay terms. In comparison, for pattern forming processes represented via reaction diffusion systems or integro-differential equations with no delays [2], the function $\mathcal{H}$ is independent of $\sigma$ and one obtains a dispersion curve representing $\sigma$ as an explicit (single branched) function of $\mathbf{k}^{1}$.

The situation that we face here is analagous to that of differential delay equations (DDEs). There has been a leap in interest in DDEs over the

\footnotetext{
${ }^{1}$ It would be possible to obtain an equation analogous to (1) for other types of steady state solutions. However, the right hand side of (1) would then include an unknown term of the form $F^{\prime}\left(u_{0}(x)\right)$.
} 
last decade following progress in making analytical representations of their solutions by exploiting the Lambert function (originally proposed 250 years ago, in 1758) [3], [5], [29]. The Lambert function $W$ is defined as any function such that

$$
W(s) e^{W(s)}=s .
$$

$W(s)$ is a complex function with an infinite number of branches. The principal branch satisfies $W_{0}(s)=s-s^{2}+O\left(s^{3}\right)$ as $s \rightarrow 0$ and can be represented by the following expression:

$$
W_{0}(s)=\sum_{n=1}^{\infty} \frac{(-n)^{n-1}}{n !} s^{n},
$$

which converges for $s<1 / e$. The other branches of the Lambert function, denoted by $W_{b}(s)$ for $b= \pm 1, \pm 2, \ldots$ are given in terms of the branches of the logarithmic function $\ln _{b}(s)=\ln (s)+2 \pi i b$ by

$$
\left.W_{b}(s)=\ln _{b}(s)-\ln \left(\ln _{b}(s)\right)\right)+A(s),
$$

where $A(s)$ is

$$
A(s)=\sum_{i=0}^{\infty} \sum_{j=1}^{\infty} K_{i j} \frac{\left(\ln \left(\ln _{b}(s)\right)\right)^{j}}{\left(\ln _{b}(s)\right)^{i+j}},
$$

and $K_{i j}$ are known constants [6].

In what follows the Lambert function will be utilised in addressing (1) since it enables us to deal with the exponential term in $\sigma$ at the cost of introducing an infinity of branches - and thereby characterising the separate branches of the resulting dispersion relation. In particular its usage allows a straightforward numerical investigation of such branches to be made separately. We discuss this in section 4 .

The fact that the complex solution of (1) (as the wave number $\mathbf{k}$ varies) results in the superposition of infinitely many curves is important within applications. It increases the pattern forming potentiality of the system, and indeed suggests that the introduction of delay effects may be critical in increasing the capacity of IDDEs to generate spatio temporal response to stimulii, transient or otherwise.

In section 2 we show how (1) arises within a specific application: that of the delay dependent Amari equation in neurodynamics [1, 8, 28]. To date, the stability and bifurcation analysis for such equations, given by Coombes and co-workers $[9,10,11]$, has considered the extreme rightward part of the resulting spectra within the complex plane (since this governs any overall loss of stability and corresponding spontaneous pattern forming process). Such 
behaviour can be rich for these equations with standing waves, bulk oscillations and travelling wave patterns dependent on the nature of the coupling kernel. Of course other spectral modes though more stable, may persist especially if these can resonate with applied stimuli (non autonomous forcing), so it may well be important within an application to characterise the features of the entire spectrum. This paper represents the first such survey for this class of IDDEs. Many of the examples included in previous works consider kernels which are symmetric around the origin and assume that local excitation is predominant. Moreover, kernels which are not peaked at the origin have already been considered in the literature, see e.g. [30]-[32]. In particular, there are papers that specifically focus on kernels that peak away from the origin e.g. [33]. However, the kernels considered here have the particular property that they are symmetric around some point $a \neq 0$ or consist of a sum of such terms. It should be emphasised that it is this property which accounts for the factor $\exp (-\varepsilon \sigma a)$ in the right hand side of (1) and thus give rise to an infinite branched spectrum.

In section 3 we reformulate (1) with use of the Lambert $W$ function and consider asymptotic approaches to the problem of determining the spectral structure; whilst in section 4 we exploit this numerically so as obtain plots for the entire spectrum of the IDDE. In section 5 we generalise the formalism developed in earlier sections to the case of systems of IDDEs and consider the case of two neural populations exciting each other. Finally, in section 6 we discuss further extensions of this work and argue why knowledge of the full spectrum is important within neurodynamical applications.

\section{The Amari time-delay neural field equation}

In this section we consider a particular example of the form of equation (1), to which the results of this paper will apply.

The description of the propagation of electrical activity in neural tissue is, in general, a difficult task. Assuming that the neural tissue is one-dimensional and that the synaptic input is a function of the pre-synaptic firing rate function the local activity of a population of neurons is described by the following Amari equation $[1,28]$

$$
u_{t}(x, t)+u(x, t)=\int_{-\infty}^{\infty} \phi(x-y) F(u(y, t-\varepsilon|x-y|)) d y, \quad t>0,
$$

where $u(x, t)$ is the synaptic activity at position $x$ and time $t, \varepsilon=1 / v$, with $v$ the velocity of propagation of the electrical activity, $\phi(x-y)$ is a real-valued smooth and bounded function which expresses the connectivity 
between points of the neural tissue and $F(u)$ is a real-valued differentiable and bounded function which is called the pre-synaptic firing rate function. It should also be noted that the generalisation of equation (5) to $n$-dimensions is immediate by replacing $x$ by $\mathbf{x}$ where $\mathbf{x} \in D \subset \mathbb{R}^{n}$.

The solutions of equation (5) include spatially and temporally periodic patterns beyond a Turing type instability, localised regions of activity such as bumps and travelling waves (see [11]-[26]). The onset of dynamic Turing instability of the homogeneous steady state has been calculated and patterns emerging from this instability have been discussed in [10]. Also, the Turing instability analysis in layered 2D systems for neural fields with spacedependent delays is treated in [27]. However, it seems that the part of the spectrum corresponding to stable modes has not been studied in any full detail. In sections 3 and 4 , we will show that the full spectrum of the neural field equation with delays has a rich structure previously undetected.

We consider uniform steady state solutions , $u=u_{0}$, where $u_{0}$ is a constant satisfying

$$
u_{0}=F\left(u_{0}\right) \phi_{0}, \quad \phi_{0}=\int_{-\infty}^{\infty} \phi(y) d y .
$$

Since the function $F(u)$ is uniformly bounded there will generically be an odd number of such solutions.

Now we write $u(x, t)-u_{0} \sim e^{\sigma(k) t+i k x}$ in equation (5) so that, up to a linear approximation, we have

$$
(\sigma(k)+1) e^{\sigma(k) t+i k x}=F^{\prime}\left(u_{0}\right) \int_{-\infty}^{\infty} \phi(x-y) e^{-\sigma(k) \varepsilon|x-y|} e^{\sigma(k) t+i k y} d y,
$$

Hence we obtain a dispersion relation

$$
\sigma(k)+1=F^{\prime}\left(u_{0}\right) \widehat{H}(k, \sigma(k))
$$

where $\widehat{H}(k, \sigma(k))$ is the Fourier transform of the function

$$
h(x, \sigma(k))=\phi(x) e^{-\varepsilon \sigma(k)|x|} .
$$

By a suitable parametrisation we can choose $F^{\prime}\left(u_{0}\right)=1$, therefore equation (8) yields

$$
\sigma+1=\widehat{H}(k, \sigma)
$$

This equation is of the form of (1), with $\mathcal{H}=\widehat{H}$, as required. Of course the exact nature of this last function depends critically upon the choice of the kernel $\phi(x)$. An important question is for which kernels $\phi(x)$, the $\sigma-$ dependence of the function $\widehat{H}(k, \sigma(k))$ includes a term of the form $e^{-\varepsilon \sigma a}$, thus 
making equation (10) a transcedental equation with infinitely many solution branches. It turns out that functions which are symmetric around a point $a \neq 0$, or which consist of a sum of such terms, yield a function $\widehat{H}(k, \sigma(k))$ of the form mentioned above. This class of functions is substantially different from many of the examples given in $[9,10,11]$ where $\phi(x)$ is dominated by a peak at the origin, making local neighbourhood behaviour the most dominant influence. In the following, in anticipation of the kernel considered in section 3 , we focus on the case that the kernel $\phi(x)$ consists of a sum of two terms, symmetric with respect to $a$ and $-a$. It is straightforward to generalise the relevant results for the case of sums consisting of an arbitrary number of terms. In particular, we consider a kernel of the form

$$
\phi(x)=\psi(x-a)+\psi(x+a), \quad a>0,
$$

where $\psi(x)$ is an even function. For simplicity, we assume that the Fourier transform of $\psi(x)$ has no poles (the case that $\widehat{\psi}(k)$ has poles can be treated similarly). Then, the function $\widehat{H}(k, \sigma)$ is given by

$$
\widehat{H}(k, \sigma)=\int_{-\infty}^{\infty} e^{-\varepsilon \sigma|x|}[\psi(x-a)+\psi(x+a)] e^{-2 \pi i k x} d x,
$$

or

$$
\widehat{H}(k, \sigma)=\int_{-\infty}^{\infty}\left[e^{-2 \pi i a(k-y)}+e^{2 \pi i a(k-y)}\right] \widehat{\psi}(k-y) \frac{2 \sigma \varepsilon}{\sigma^{2} \varepsilon^{2}+4 \pi^{2} y^{2}} d y .
$$

Using the residue theorem to evaluate the integral of the right hand side, we obtain

$$
\widehat{H}(k, \sigma)=e^{-\varepsilon \sigma a}\left[e^{-2 \pi i a k} \widehat{\psi}\left(k-i \frac{\sigma \varepsilon}{2 \pi}\right)+e^{2 \pi i a k} \widehat{\psi}\left(k+i \frac{\sigma \varepsilon}{2 \pi}\right)\right],
$$

where the term $e^{-\varepsilon \sigma a}$ appears explicitly.

For functions of the form of equation (11), where $\psi(x)$ is an even function and $a>0$, the dispersion relation, namely equation (10) (or equivalently (1)), is transcendental. We will therefore obtain an infinite number of solution branches. On the other hand if $\phi$ is such that $\widehat{H}(k, \sigma)$ is a rational function of $\sigma$, then (1) will have only a finite number of branches; since then we have a polynomial, with $k$ dependent coefficients, to be solved for $\sigma$. Hence the inclusion of time delays (in the case of the neural field equation due to the speed of propagation) is a necessary but not a sufficient condition for the spectrum to contain an infinite number of branches. For example, for any $\phi(x)$ of the form

$$
\phi(x)=|x|^{n} e^{-r|x|}
$$


where $n \in \mathbf{N}, r>0$, there are only finitely many branches whatever the value of $\varepsilon>0$. Indeed, for such a kernel

$$
\widehat{H}(k, \sigma)=\frac{n !}{(\varepsilon \sigma+r+2 \pi i k)^{n+1}}
$$

This may seem counterintuitive to some who might expect the infinite degrees of freedom in any differential delay equation to correspond to an infinite number of branches for IDDEs regardless of the choice of kernel. However, it should be emphasised that the choice of kernel has a key role to play: any kernel of the class defined by equation (15) is strongly dominated by its local behaviour at the origin, thus it cannot yield an infinite number of spectral branches. On the other hand, replacing $x$ by $x-a, a \neq 0$, in (15), we find

$$
\widehat{H}(k, \sigma)=e^{-\varepsilon \sigma a} e^{-2 \pi i k a} \frac{n !}{(\varepsilon \sigma+r+2 \pi i k)^{n+1}},
$$

where the term $e^{-\varepsilon \sigma a}$ appears naturally. This term is what renders the dispersion relation (10) transcedental and results in a spectrum with an infinite number of branches.

In the next section, motivated by the above discussion, we consider a kernel obtained from equation (11) by replacing $\psi(x-a)$ with $\psi(x-a, r)$ where $\psi(x, r)=\frac{r}{2 e^{r|x|}}$ and $a=1$. Therefore, we choose $\phi(x)$ to be even with peaks at a fixed unit distance from the origin, reflecting the case where the major excitory influences are those that travel from a unit distance away and are thus delayed.

\section{Spectra involving an infinite multiplicity of branches}

Consider the kernel $\phi(x)$ given by

$$
\phi(x, r)=\frac{r}{2 e^{r|x-1|}}+\frac{r}{2 e^{r|x+1|}},
$$

which expresses a dominance of a nonlocal interaction from a unit distance away in both directions.

Then, for $\operatorname{Re}(r+\varepsilon \sigma)>0$, equation (10) becomes

$$
\sigma+1=\frac{e^{-\varepsilon \sigma}\left(1+e^{-2 r}\right) r[(r+\varepsilon \sigma) \cos (2 k \pi)-2 k \pi \sin (2 k \pi)]}{4 k^{2} \pi^{2}+(r+\varepsilon \sigma)^{2}} .
$$

First, we consider $k$ fixed and show that there is an infinity of values for $\sigma$ corresponding to each value of $k$ : 
Multiplying both sides of equation (19) by $\varepsilon e^{\varepsilon(\sigma+1)}$, we find an equation where the dependence of the right hand side on $\sigma$ is only rational, namely

$$
\varepsilon(\sigma+1) e^{\varepsilon(\sigma+1)}=\frac{\varepsilon e^{\varepsilon}\left(1+e^{-2 r}\right) r[(r+\varepsilon \sigma) \cos (2 k \pi)-2 k \pi \sin (2 k \pi)]}{4 k^{2} \pi^{2}+(r+\varepsilon \sigma)^{2}} .
$$

Using the definition of the Lambert function $W(s)$ given by equation (2), we find the following expression for the spectral values

$$
\sigma=\frac{1}{\varepsilon} W(R(k, r, \sigma))-1,
$$

where the function $R(k, r, \sigma)$ is given by the right hand side of equation (20).

Equation (21) and the fixed point theorem imply that for each branch of the Lambert function there is a corresponding value of $\sigma$. Since the Lambert function has infinitely many branches, there is an infinity of spectral values $\sigma$.

In the following, we consider some important limiting cases:

\section{The limit $r \rightarrow \infty$}

In the limit $r \rightarrow \infty$, equation (21) becomes

$$
\sigma=\frac{1}{\varepsilon} W\left(\varepsilon e^{\varepsilon} \cos (2 k \pi)\right)-1 .
$$

Comparing equation (22) with equation (26) we find that these two equations are the same if $\beta=1$ and $\lambda=\cos (2 k \pi)$. Therefore, for the extreme values of $\lambda= \pm 1,\left(k=1, \pm 1, \pm 2, \ldots\right.$ and $\left.k= \pm \frac{1}{2}, \pm \frac{3}{2}, \ldots\right)$, the relevant spectrum is given by the green and pink points of figure 1 (where wlog $\varepsilon=1$ ).

This result should come as no surprise since as $r \rightarrow \infty$, the kernel (18) becomes a sum of two delta functions at $x= \pm 1$ describing an infinite chain of neurons connected to their nearest neighbours. These coupled neurons can also be described by following system:

$$
\begin{aligned}
& u_{1 t}(t)+u_{1}(t)=\mu u_{2}(t-\varepsilon), \\
& u_{2 t}(t)+u_{2}(t)=\mu u_{1}(t-\varepsilon) .
\end{aligned}
$$

Then, letting $u(t)=\left(u_{1}(t), u_{2}(t)\right)(1, \pm 1)^{T}$, the above system reduces immediately to the equation

$$
u_{t}(t)+\beta u(t)=\lambda u(t-\varepsilon)
$$


where $\beta, \lambda$ and $\varepsilon>0$ are constants, $\mu= \pm \lambda$ and spectrum depicted in figure 1 .

Inserting $u(t) \sim e^{\sigma t}$ we obtain the characteristic equation for the spectral values:

$$
\sigma+\beta=\lambda e^{-\varepsilon \sigma},
$$

which is analogous to (1). It follows that solutions of equation (25) must satisfy

$$
\varepsilon(\sigma+\beta)=W\left(\varepsilon \lambda e^{\varepsilon \beta}\right),
$$

and there is an infinite number of eigenmodes associated with equation (25). These modes are given in terms of the branches of the Lambert function by the expression

$$
\sigma=\frac{1}{\varepsilon} W_{b}\left(\varepsilon \lambda e^{\varepsilon \beta}\right)-\beta
$$

For example, the first few values within the complex $\sigma$-plane are shown in figure 1 for $\lambda$ both positive and negative.

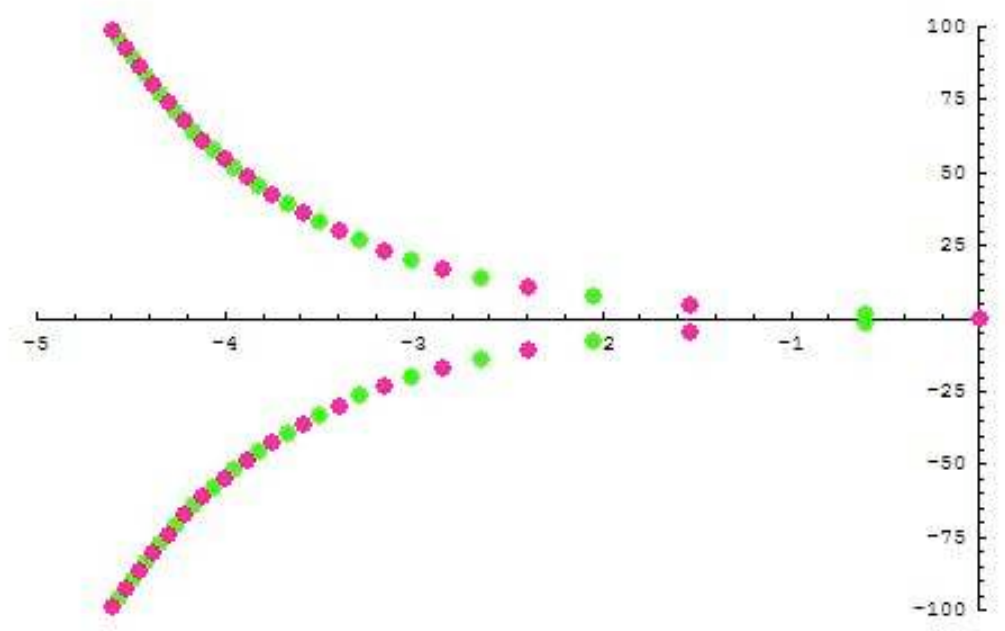

Figure 1. Spectrum when $\varepsilon=\beta=1$ and $\lambda=1$ (pink) and -1 (green).

\section{The limit $\varepsilon \rightarrow 0$}

Assuming that $\varepsilon \rightarrow 0$, namely that there is no delay, equation (19) becomes

$$
\sigma+1=\frac{\left(1+e^{-2 r}\right)\left[r^{2} \cos (2 k \pi)-2 r k \pi \sin (2 k \pi)\right]}{4 k^{2} \pi^{2}+r^{2}} .
$$


Hence, there exists only a single real mode for a fixed $k$ (and $r$ ), corresponding to the limit of the principal branch for the Lambert function: the other branches have escaped to minus infinity. Taking the limit $r \rightarrow \infty$, in (27), we obtain

$$
\sigma+1=\cos (2 k \pi)
$$

\section{The numerical determination of the spec- trum}

Here we illustrate how to determine numerically the full spectrum of equation (19) as a particular example of equation (1).

We proceed as follows. First, setting $k=0$ in (21), we obtain

$$
\sigma=\frac{1}{\varepsilon} W_{b}\left(\frac{\varepsilon e^{\varepsilon \sigma}\left(1+e^{-2 r}\right) r}{(r+\varepsilon \sigma)}\right)-1
$$

where $W_{b}$ are the branches of the Lambert function given by (3) for $b=0$, and (4) for $b= \pm 1, \pm 2, \ldots$ with $A(s)=0$. We use this equation to iterate to a single point solution on each succesive branch of the Lambert function, thus ensuring that we commence each seperate branch of the spectrum from a unique point. Then in summary, to solve equation (19), we start out from each solution branch (parametrised as "branch $b$ "), at $k=0$, and we vary $k$ incrementally, each time using the previous solution as the starting point for a standard Newton iteration for $\sigma$. This results in the computation of a single branch. Each branch is even in $k$ and approaches infinity as $|k| \rightarrow \infty$. In figures 2 and 3, we show seven such branches. The principal branch ("branch 0 ") corresponds to the part of the spectrum that is real. 

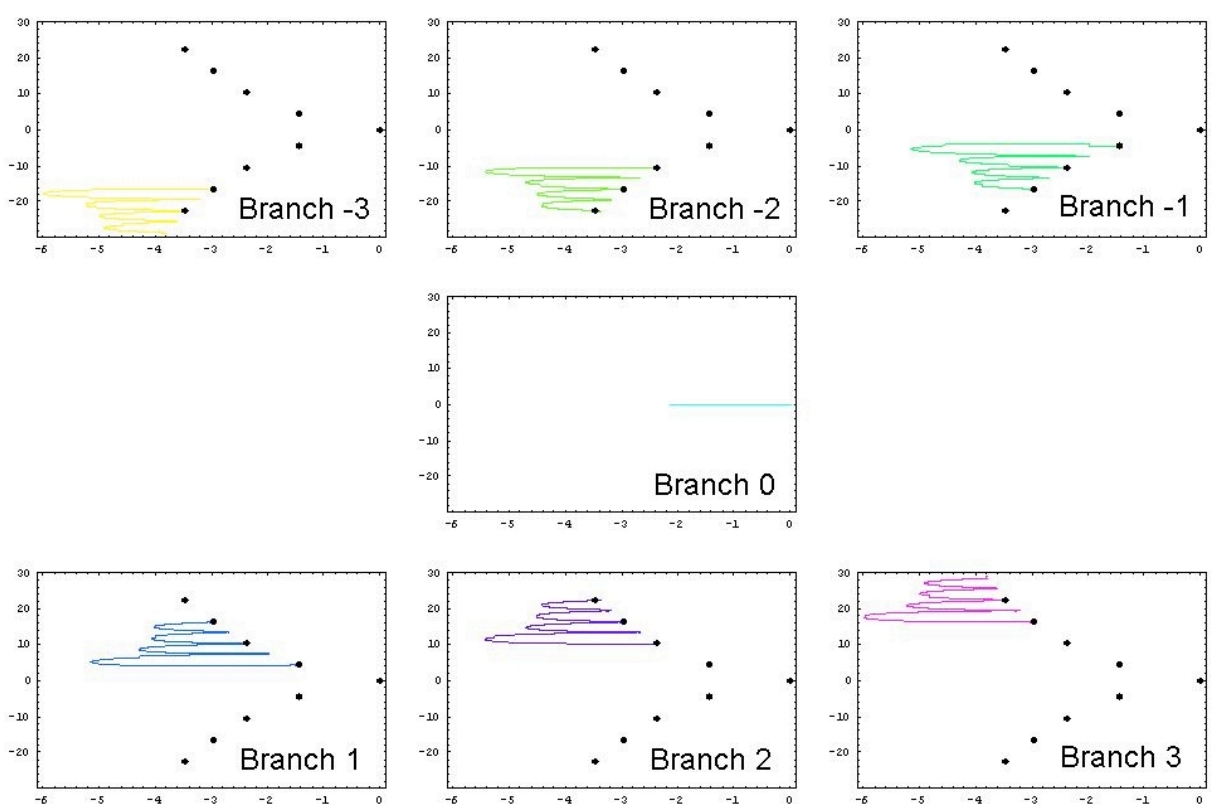

Figure 2. Separate spectral branches $(b=0, \pm 1, \pm 2, \pm 3)$, each starting (when $k=0$ ) at a separate point (shown as bold) generated via the $b$ th branch of the Lambert function, before looping through successive points.

Here $\varepsilon=1$ and $r=20$.

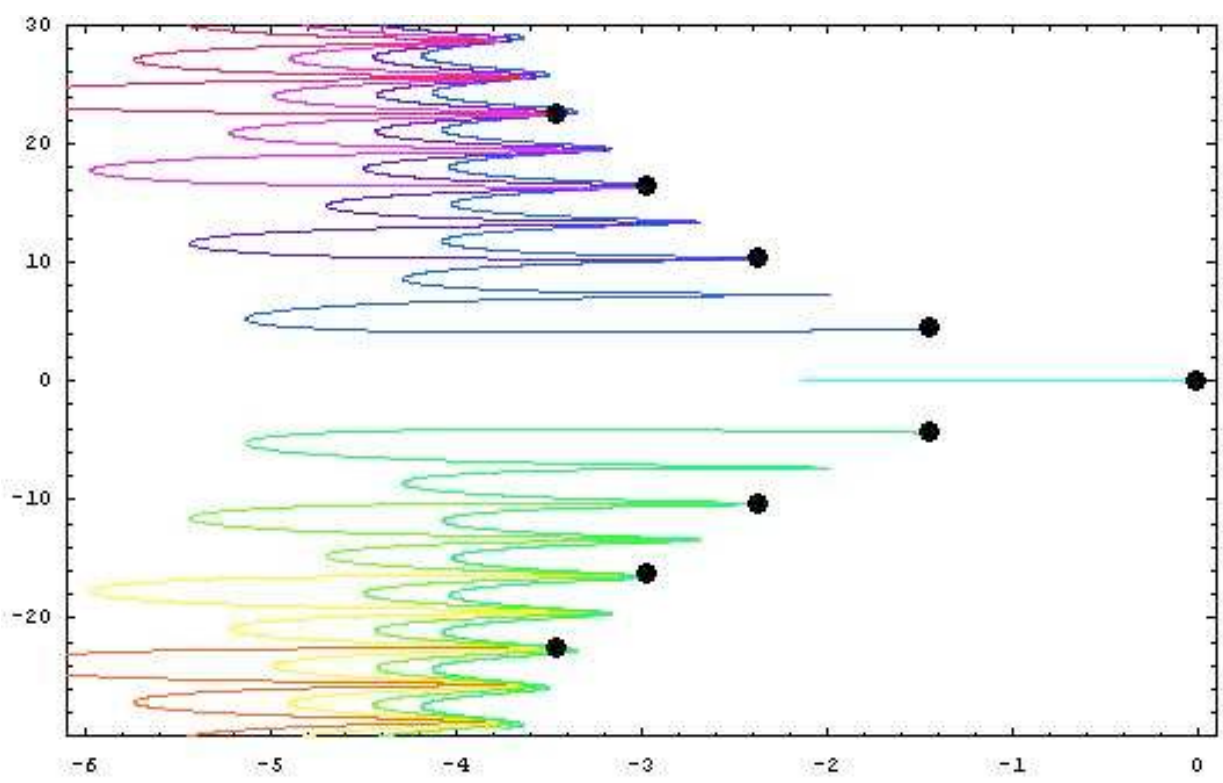

Figure 3. Superposition of the seven branches of the spectra in Figure 2. Here $\varepsilon=1$ and $r=20$.

As $\varepsilon \rightarrow 0$ all of branches except the principal branch move off to infinity parallel to the negative real axis, leaving the principal branch to become the 
remaining real spectrum $\sigma=-1+\hat{\phi}(k)$. As $r$ tends to infinity the "loops" in the spectrum become longer, with each loop approaching two parallel lines at constant imaginary values from the point at infinity, see figure 4 . Finally, when $\varepsilon$ becomes large the real part of the spectrum is lost, see figure 5 .

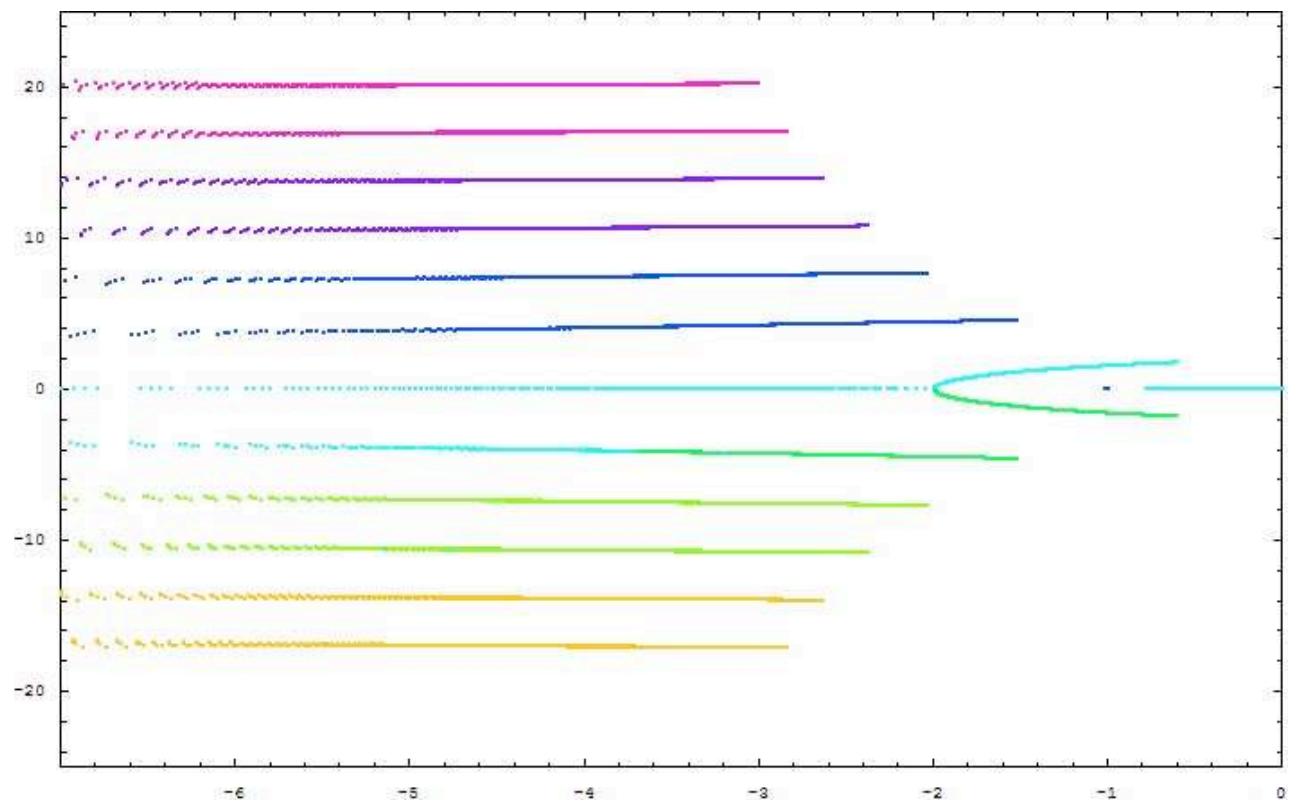

Figure 4. The spectra of the Amari equation for $\varepsilon=1$ and $r=200$.

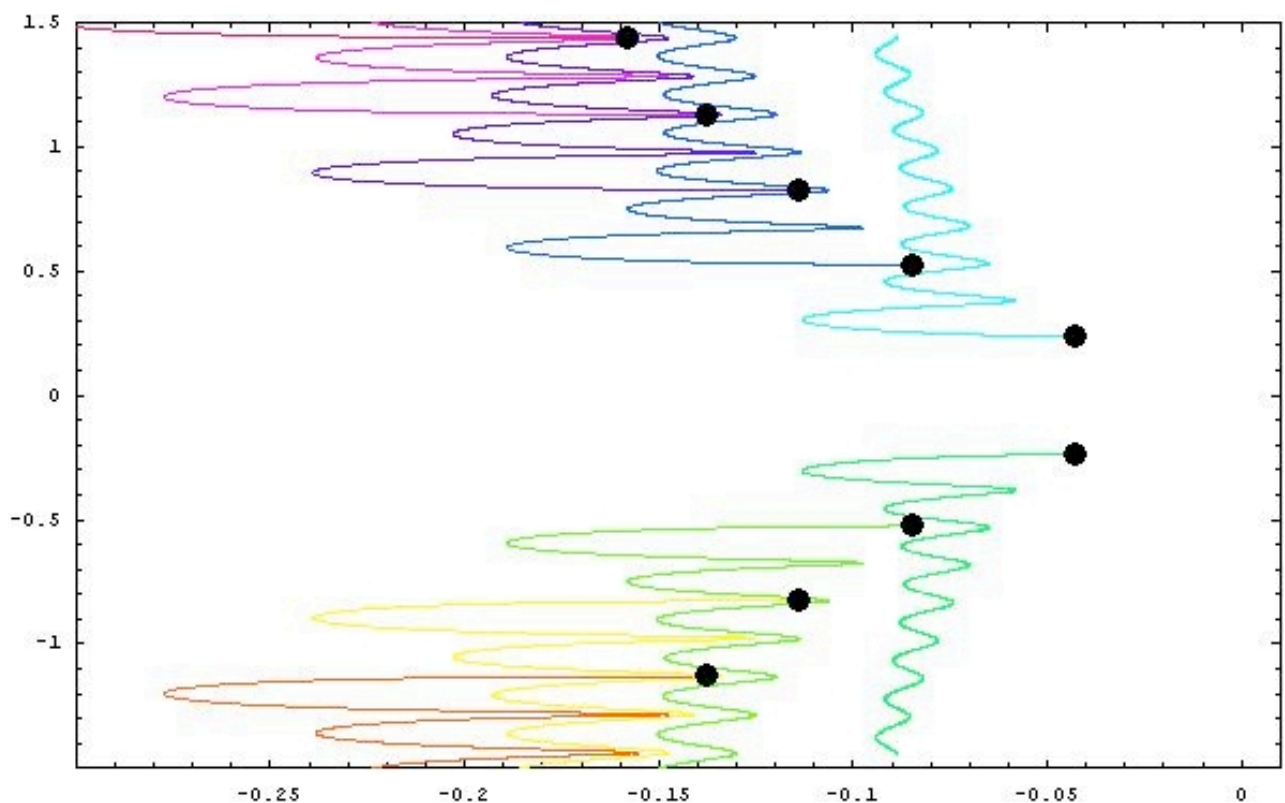

Figure 5. The spectra for $\varepsilon=20$ and $r=2$.

Let us now show one further example. Here the kernel $\phi(x)$ contains a sum of four terms, similar to the terms appearing in (18), each symmetric 
about points $x \pm 1$ and $x= \pm 2$ respectively, which we expect to resonate with each other. Moreover, choosing the amplitude of the terms appropriately it is possible to move many of the spectral cusps into the right hand side of the complex plane. As they do so, there will be Turing-Hopf type instabilities at the corresponding points $\sigma(k)$, which are purely imaginary and can have non zero as well as zero values for $k$ (giving birth to periodic travelling wave patterns and bulk oscillatory patterns).

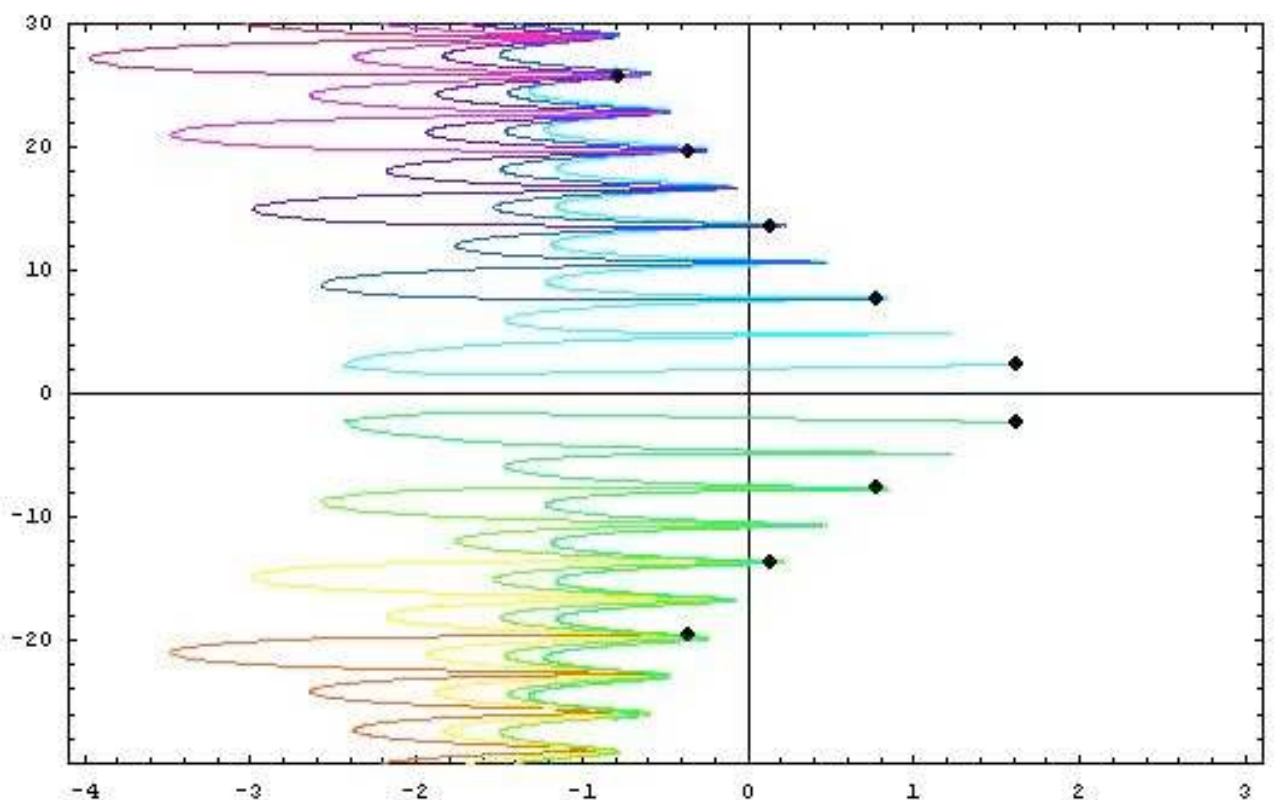

Figure 6. Spectra involving cusps in the right hand side of the complex plane.

\section{Systems}

So far we have considered (1) and have shown how it might arise from an equation with a single state variable, $u(x, t)$. Here we shall generalise the formulation developed in earlier sections to consider systems.

Let $\mathbf{u}(x, t)=\left(u_{1}(x, t), \ldots, u_{m}(x, t)\right)^{T}$ be a $\mathbb{R}^{m}$ valued state variable satisfying

$$
\mathbf{u}_{t}(x, t)+A \cdot \mathbf{u}(x, t)=\int_{-\infty}^{\infty} \Phi(x-y) \cdot \mathbf{F}(\mathbf{u}(y, t-\varepsilon|x-y|)) d y, t>0
$$

Here, '?' denotes matrix multiplication and $\mathbf{F}: \mathbb{R}^{m} \rightarrow \mathbb{R}^{m}$ is a nonlinear mapping (Lipschitz continuous to guarantee local existence); $A$ is a $m \times m$ 
constant matrix, describing the point dynamics and $\Phi(x)$ is an $m \times m$ matrixvalued smooth, integrable kernel.

We set $\Phi_{0}=\int_{-\infty}^{\infty} \Phi(x) d x$, and assume that $\mathbf{u}=\mathbf{u}_{0}$ is a constant steady state, satifying

$$
A \cdot \mathbf{u}_{0}=\Phi_{0} \cdot \mathbf{F}\left(\mathbf{u}_{0}\right) .
$$

Linearising about $\mathbf{u}_{0}$, we write

$$
\mathbf{u}(x, t)-\mathbf{u}_{0} \sim e^{\sigma(k) t+i k x} \mathbf{v}(k),
$$

so that

$$
(\sigma(k) I+A) \cdot \mathbf{v}(k)=\int \Phi(x-y) \cdot d \mathbf{F} \cdot e^{-\sigma(k) \varepsilon|x-y|} e^{i k(y-x)} d y \cdot \mathbf{v}(k) .
$$

Here $d \mathbf{F}=d \mathbf{F}\left(\mathbf{u}_{0}\right)$ is the Jacobian of $\mathbf{F}$ at $\mathbf{u}_{0}$.

Define the integral operator $\hat{\mathbf{H}}(k, \sigma(k))$ to be the (matrix-valued) Fourier transform of $\Phi(x) \cdot d \mathbf{F} \cdot e^{-\sigma(k) \varepsilon|x|}$. Then we have

$$
(\sigma(k) I+A) \cdot \mathbf{v}(k)=\hat{\mathbf{H}}(k, \sigma(k)) \cdot \mathbf{v}(k) .
$$

Thus the spectrum is given by

$$
\operatorname{det}(\sigma(k) I+A-\hat{\mathbf{H}}(k, \sigma(k)))=0 .
$$

Let us consider a more specific example with $m=2$. Take

$$
A=\left(\begin{array}{cc}
a_{1} & 0 \\
0 & a_{2}
\end{array}\right), \quad \Phi=\left(\begin{array}{cc}
\phi_{1}(x) & 0 \\
0 & \phi_{2}(x)
\end{array}\right), \quad d \mathbf{F}\left(\mathbf{u}_{0}\right)=\left(\begin{array}{cc}
0 & \beta_{1} \\
\beta_{2} & 0
\end{array}\right) .
$$

Let

$$
\widehat{H}_{j}(k, \sigma)=\int_{-\infty}^{\infty} e^{-2 \pi i k x} \phi_{j}(x) e^{-\varepsilon \sigma|x|} d x
$$

Assuming that $\widehat{H}_{j}(k, \sigma)=e^{-\varepsilon \sigma} R_{j}(k, \sigma)$, where $R_{j}(k)$ is a polynomial in $\sigma$, equation (31) can be written as

$$
\operatorname{det}\left(\begin{array}{ll}
\sigma+a_{1} & -\beta_{1} e^{-\varepsilon \sigma} R_{1}(k, \sigma) \\
-\beta_{2} e^{-\varepsilon \sigma} R_{2}(k, \sigma) & \sigma+a_{2}
\end{array}\right)=0
$$

So the spectrum is determined by solving the characteristic equation

$$
\left(\sigma+a_{1}\right)\left(\sigma+a_{2}\right)=\beta_{1} \beta_{2} e^{-2 \varepsilon \sigma} R_{1}(k, \sigma) R_{2}(k, \sigma) .
$$

Now if $a_{1}=a_{2}=1$, say, equation (33) reduces to

$$
\sigma+1=\mu \sqrt{\beta_{1} \beta_{2}} e^{-\varepsilon \sigma}\left(R_{1} R_{2}\right)^{1 / 2}(k, \sigma), \quad \mu= \pm 1 .
$$


The last equation yields

$$
\sigma=\frac{1}{\varepsilon} W\left(\mu \sqrt{\beta_{1} \beta_{2}} \varepsilon e^{\varepsilon a}\left(R_{1} R_{2}\right)^{1 / 2}(k, \sigma)\right)-1 .
$$

For example, if $\phi_{1}$ and $\phi_{2}$ are both given by the kernel (18), we obtain the figure below, showing the multi-branched spectrum in the case where $\mu= \pm 1$, corresponding to the purple and green curves respectively. Again, the bold points represent the positions where the wave number $k$ is zero; and there are many crossing points where the same value for $\sigma$ corresponds to distinct values of $k$. These values of $k$ are on distinct branches, which we have tracked by starting out on separate branches of the Lambert function.
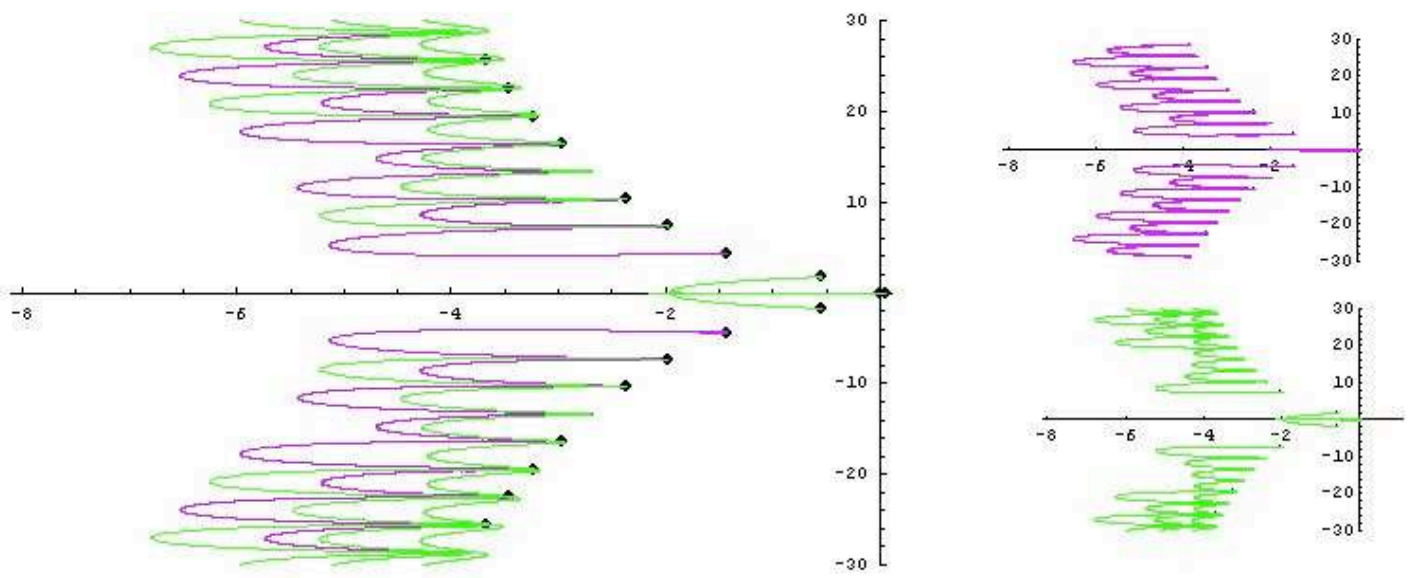

Figure 7 . The parts of the branched spectrum for $\mu=1$, top right; $\mu=-1$, bottom right; and together on the left.

\section{Resonance and input-output response}

In this section we illustrate why a detailed knowledge of the spectrum, arising from the linearization of an IDD system about a stable restpoint, is so functionally important within applications such as neurodynanmics.

Systems such as the neurodynamical Amari model are capable of showing spontaneous, instability driven pattern formation. An example of such a situation is epilepsy which is dominated by internally driven patterning without any stimulii. However, this is an aberant state of affairs for neurodynamical systems in vivo; a much more normal situation involves the responses of a stable neurodynamical system to a range of localised incoming signals from sensory mechanisms or other parts of the processing aparatus up stream. 
Furthermore, spontaneous patterns of just a few types simply do not provide a large enough lexicon for a necessary plethora of cognitive functions.

Therefore we suggest that: (i) the Amari system should be conceived as an input-response unit, with the addition of a forcing (input term) ${ }^{2}$ and (ii) for neurodynamics it is real time pattern response, at zero computational cost, that is highly desirable, nay necessary: yet to date it has not been previously discussed or illustrated.

Consequently we must ask for which stimuli can the Amari system produce a coherent response, synchronising in time with the input, and displaying a suitable pattern over space?

The knowledge of the spectrum gained in previous sections is precisely what we need to address this question. Indeed, the input-response is multifaceted, in analogy with the resonant harmonics of a mechanical system. The successive forays of the spectrum towards the imaginary axis provide a capacity for distinctive types of responses to correspondingly distinct types of stimulii. This capacity would be absent without them.

Typically we expect that we will be in a situation where a neurodynamical system will be stable one, close to a resting point, with spectrum characterised by $\operatorname{Re}(\sigma) \leq 0$. Then, any solution decays to zero (the equilibrium) for large time regardless of its initial history; and could in principal be written as a linear combination of all the decaying modes.

Suppose next that such a system is stimulated by applying an external excitory term, proportional to $e^{i(\omega t+k x)}$, for some $\omega>0$ and real $k$. All such excitations could be written as a suitable integral over $k$ of terms involving exponentials. Therefore the solution of the resulting inhomogeneous system can be also written as a linear combination of $e^{i(\omega t+k x)}$ terms as well as decaying modes. This is fine except when one of the parts of the spectrum $\sigma(k)$ gets near to the imaginary axis, say at $i \omega$. Then a familar resonance phenomenon kicks in.

The long term solution of the IDDE contains a term that is of the form

$$
\frac{e^{i(\omega t+k x)}}{i \omega+1-\widehat{H}(k, \omega)} \text {. }
$$

The denominator which is equal to the inverse of the response amplitude becomes closest to zero and the relative response to the stimulus is maximised precisely where the pairs $(k, \omega)$ are closest to some part of the spectrum $(k, \sigma(k))$ within the left hand side of the plane.

In this sense, the Amari system acts like a filter: it has the ability to recognise and respond whenever it is excited in such a way as to almost res-

\footnotetext{
${ }^{2}$ Similarly to the analysis of [34]
} 
onate with one or more of its rightward modes (where the real part of $\sigma(k)$ has a local maximum): it relatively amplifies such inputs, and dampens others (see Figure 8 below). This is "resonance" in action; the system recognises certain inputs and ignores others at no computational cost, in real time. We contend that it is these "hot spot" resonant modes that are the currency of input-output response: the latter being a dynamic and spatial distribution of neural activity. It should be emphasised that it is the delays in the Amari IDDE that produce this multiple resonance, or "harmonic" behaviour. This behaviour in turn, increases the capacity of the system to show a muliplicity of responses.

Therefore, the answer to the question raised above could be briefly stated as follows: modes of increased responsiveness of the neurodynamical system correspond to the forays of the real part of the spectrum of the Amari system.

In summary, the structure of the spectrum for the IDDE is (i) intimately connected with the choice of the kernel and (ii) a crucial component in filtering input information and producing a discrete range of resonant responses, as opposed to a passive continuum response. Of course, the nonlinearities become important away from equilibrium in the longer term. Nevertheless, for natural neurodynamical systems to perform rapid coherent signal "recognition and response" behaviour it is the complex nature of the spectra derived in this paper, even for simple systems, that is exactly what is required.

In practice, observations of neurodynamical patterns and waves via scans will allow us constrain and locate kernel behavioural properties at the meso level within the brain. Anisotropy, spatial variability, behaviour at boundaries, and piecewise continuity will make the future inverse and forward problems much harder. However, we suggest that such a programme cannot commence without a solid understanding of the rich spectral structure that is available, even for the ideal (spatially uniform, isotropic) situation considered here. 
requires no computation and responds in real time). This idea suggests that in seeking to understand reasoning processes from patterns and connectivities within single fMRI scans [35] we are looking in the wrong place: we must see the evolution of such activity over time as the response to the upstream stimuli.

Acknowledgement. The authors acknowledge support from EPSRC Bridging the Gaps 'Cognitive Systems Science' Grant No. EP/F033036/1.

\section{References}

[1] S I Amari, Dynamics of pattern formation in lateral inhibition type neural fields, Biological Cybernetics, 27, 77-87, (1977).

[2] P. Grindrod, Patterns and Waves: The Theory and Applications of Reaction-Diffusion Equations, Second Edition, OUP (1995).

[3] R M Corless, G H Gonnet, D E G Hare, D J Jeffrey and D E Knuth, On the Lambert $\mathrm{W}$ function, Advances in Computational Mathematics, 5 329?359, (1996).

[4] D Henry, Geometric Theory of Semilinear Parabolic Equations, Lecture notes in Mathematics, Springer (1981)

[5] J H Lambert, Observations variae in Mathesin Puram., Acta Helvitica, physico-mathematico-anatomico-botanico-medica 3, 128-168, (1758).

[6] N G De Bruijn, Asymptotic Methods in Analysis, North Holland (1961)

[7] D Henry, Geometric Theory of Semilinear Parabolic Equations, Lecture notes in Mathematics, Springer (1981)

[8] Beim Graben, P. and Potthast, R.: "Inverse Problems in Neural Field Theory", SIAM J. Appl. Dyn. Systems, accepted for publication

[9] S Coombes, GJ Lord and MR Owen, Waves and bumps in neuronal networks with axo-dendritic synaptic interactions, Physica D, 178, 219241 (2008)

[10] N A Venkov, S Coombes and PC Matthews, Dynamic instabilities in scalar neural field equations with space-dependent delays, Physica D, 232, 1-15 (2007)

[11] S Coombes, Waves, bumps, and patterns in neural field theories, Biological Cybernetics, 93, 91-108 (2005) 
[12] P Bressloff and S. E. Folias, Front bifurcations in an excitatory neural network. SIAM j. Appl. Math., 65, 131151, 2004.

[13] P. C. Bressloff, Bloch waves, periodic feature maps, and cortical pattern formation. Phys. Rev. Lett., 89, 2002.

[14] S. Coombes and M. R. Owen. Bumps, breathers, and waves in a neural network with spike frequency adaptation. Phys. Rev. Lett., 94 148102, (2005).

[15] Y. Guo and C.C. Chow. Existence and stability of standing pulses in neural networks: I. existence. SIAM J. Appl. Dyn. Syst., 4 217248, (2005)

[16] Y. Guo and C.C. Chow. Existence and stability of standing pulses in neural networks: II. stability. SIAM J. Appl. Dyn. Syst., 4 249281, (2005)

[17] C. R. Laing and W. C. Troy. Two-bump solutions of Amari type models of working memory. Phys. D, 178 190218, (2003)

[18] C. R. Laing, w. C. Troy, B Gutkin, and G. B Ermentrout. Multiple bumps in a neuronal model of working memory. SIAM J. of Applied Math., 63, no.1:6297, (2002)

[19] C. R. Laing and William C. Troy. PDE methods for nonlocal models. SIAM J Appl. Dyn. Syst., 2 487516, (2001)

[20] J. D. Pinto and G. B. Ermentrout. Spatially structured activity in synaptically coupled neuronal networks: 1 traveling fronts and pulses. SIAM J. Appl. Math., 62 206225, (2002)

[21] J. D. Pinto and G. B. Ermentrout. Spatially structured activity in synaptically coupled neuronal networks:2 lateral inhibition and standing pulses. SIAM J. Appl. Math., 62 226243, (2002)

[22] J. D. Pinto, K. R. Jackson, and C. E. Wayne. Existence and stability of traveling pulses in a continuous neuronal network. SIAM J. Appl. Dynam. Syst., 4 954984, (2005)

[23] J. E. Rubin, D. Terman, and C. C. Chow. Localized bumps of activity sustained by inhibition in a two-layer thalamic network. J. Comp. Neurosci., 10 313331, (2001) 
[24] J. E Rubin and W. C Troy. Sustained spatial patterns of activity in neuronal populations with or without lateral inhibition. SIAM J. Appl. Math., 84, 5, 1809(2004)

[25] B. Sandstede. Evens functions and nonlinear stability of traveling waves in neuronal network models. International J. of Bifurcation and Chaos, 17 26932704, (2007)

[26] L. Zhang. On stability of traveling wave solutions in synaptically coupled neuronal networks. Diff. and Inte. Equ., 16 513536, (2003)

[27] S Coombes, N A Venkov, L Shiau, I Bojak, D T J Liley and C R Laing, Modeling electrocortical activity through improved local approximations of integral neural field equations, Physical Review E, 76, 051901-8 (2007)

[28] HR Wilson and JD Cowan, A mathematical theory of the functional dynamics of cortical and thalamic nervous tissue, Kybernetik, 13, 55-80 (1973)

[29] F Asl and A G Ulsoy, Analysis of a System of Linear Delay Differential Equations, J. Dynamic Systems, Measurement, and Control, 125, 215$223(2003)$

[30] W C Troy, Traveling Waves and Synchrony in an Excitable Large-Scale Neuronal Network with Asymmetric Connections, SIAM J. Appl. Dyn. Syst., 7, 1247 (2008)

[31] A. Hutt and F. M. Atay, Analysis of nonlocal neural fields for both general and gamma-distributed connectivities, Phys. D, 203, 30 (2005)

[32] F. M. Atay and A. Hutt, Stability and bifurcations in neural fields with finite propagation speed and general connectivity, SIAM J. Appl. Math. 65, 2, $644(2005)$

[33] J. Rinzel, D. Terman, X.-J. Wang, and B. Ermentrout, Propagating Activity Patterns in Large-Scale Inhibitory Neuronal Networks, Science, 279, 1351 (1998)

[34] A. Hutt and F. M. Atay, Spontaneous and evoked activity in extended neural populations with gamma-distributed spatial interactions and transmission delay, Chaos, Solitons Fractals, 32, 547 (2007)

[35] R A Poldrack, The role of fMRI in Cognitive Neuroscience: where do we stand?, Curr. Opin. in Neurobiol., 18, 223-227 (2008) 


\title{
On the spectra of certain Integro-Differential-Delay problems with applications in neurodynamics
}

\author{
Peter Grindrod * and Dimitris Pinotsis *†
}

May 4, 2010

\begin{abstract}
We investigate the spectrum of certain Integro-Differential-Delay equations (IDDEs) which arise naturally within spatially distributed, nonlocal, pattern formation problems. Our approach is based on the reformulation of the relevant dispersion relations with the use of the Lambert function. As a particular application of this approach, we consider the case of the Amari delay neural field equation which describes the local activity of a population of neurons taking into consideration the finite propagation speed of the electric signal. We show that if the kernel appearing in this equation is symmetric around some point $a \neq 0$ or consists of a sum of such terms, then the relevant dispersion relation yields spectra with an infinite number of branches, as opposed to finite sets of eigenvalues considered in previous works. Also, in earlier works the focus has been on the most rightward part of the spectrum and the possibility of an instability driven pattern formation. Here, we numerically survey the structure of the entire spectra and argue that a detailed knowledge of this structure is important within neurodynamical applications. Indeed, the Amari IDDE acts as a filter with the ability to recognise and respond whenever it is excited in such a way so as to resonate with one of its rightward modes, thereby amplifying such inputs and dampening others. Finally, we discuss how these results can be generalised to the case of systems of IDDEs.
\end{abstract}

\footnotetext{
${ }^{*}$ Department of Mathematics and the Centre for Integrative Neuroscience and Neurodynamics, University of Reading, UK

${ }^{\dagger}$ Present address: Wellcome Trust Centre for Neuroimaging, University College London, UK
} 